\title{
Seeking universals
}

\section{Melvin Konner}

$\mathrm{D}$ espite the successes of behavioural biology, some still deny that human nature exists. It is difficult to understand what this denial means. Surely such features as, say, bipedal walking, language and a dependence on eight amino acids are integral to our being? Not necessarily. People who cannot walk or talk are still human. Phenylalanine is poisonous to some people. Many other species need those amino acids. And the enormous variety of human languages would seem to be the final blow.

Actually these answers, valid in themselves, are irrelevant, little more than a debater's move. The debate becomes especially fierce and the evasion especially deft when behaviour is discussed, particularly if the behaviour is deemed undesirable - gluttony, lust or violence. The real issue is easily drawn: are some things in human behaviour strongly and inherently part of what we are? As with nucleic-acid sequences and skeletal morphology, some features vary widely within the species, some little or not at all.

The genome sequencers have identified features that we all share. Only 1 or $2 \%$ are unique to us, but all are part of our nature.

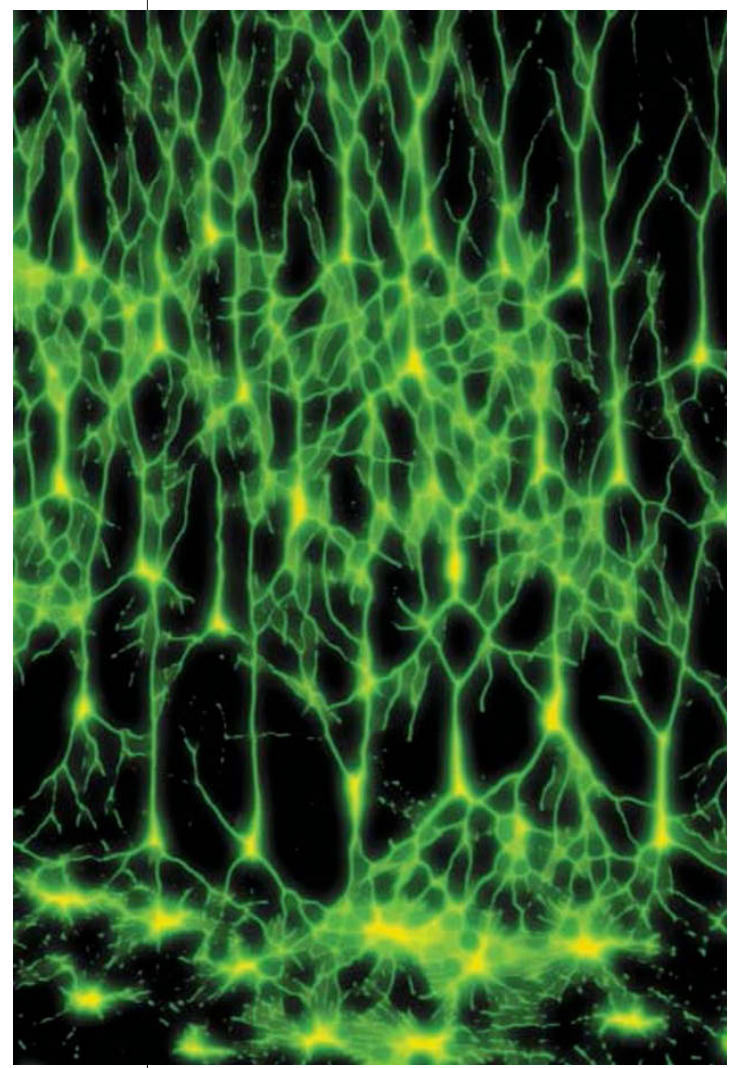

Innate nature: are some so-called cultural phenomena wired in the brain, even before birth?
Characterizing human behaviour, emotion and mind can be much less expensive and at least equally successful. As with the genome or the skeleton, neither the absence of perfect invariance nor an extension to other species prevents a feature from helping us to define our humanity. In fact, a behaviour can be far from universal and still shed light on our nature. As Joseph Greenberg pointed out, any feature of phonology or syntax that is not distributed randomly over the world's languages must be guided by a bias, which needs explaining. The bias is in the nature of language and is therefore in human nature.

Not all of human behaviour lends itself to such rigorous analysis as phonology and syntax, but it can be studied. The search for universals is a good initial step. Where variation ends, human nature begins; where variation is systematic rather than random, the biaseslead to laws of human nature. However, even 'universal' needs parsing. It can mean that every normal member of the species has it, as with bipedal walking or language. But it can also mean that every member of an age or sex class exhibits it, as with the Moro reflex of newborn infants or the ejaculatory sequence of postpubertal males. It can mean just that some members of every population show it; thus, paradoxically, it is universal despite being unusual, as with depression or homicide. It can be cultural, as with incest prohibitions, marriage or the mastery of fire. And finally, far from being rigid, what is universal may be only the pattern of variation, as in the three- or five-factor models of personality.

Where do such biases come from? Ultimately they come from the genome, through molecular function and anatomical structure, especially in the neural and endocrine systems. But many variables can intervene, and we must be careful about unknowns in the causal chain. The division of labour by sex once seemed to be universal; now it is largely superseded by cultural and technological development. The human genome generates a division of labour by sex in certain circumstances: high infant and child mortality; limitations of suitable weaning foods; the need for women to be pregnant or lactating; and the value of physical strength for hunting, largeanimal husbandry and pre-modern war.

But change those circumstances and the 'universal' disappears. Consider smiling in greeting, a feature shown by all normal humans over eight weeks old — or, more precisely, over forty-eight weeks of conceptual age. This stronger link to conceptual than postnatal age strengthens the case for smiling in greeting as a feature of human nature, because it suggests a genetically guided process of neural development rather than a

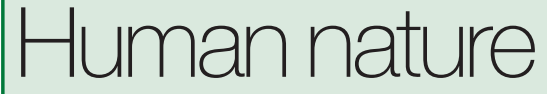

In the era of genomics and brain

imaging, hypotheses about human

nature are more testable than ever.

specified dose of experience. In fact, experience may have a modest role; socially deprived or blind infants smile with small delays. In adult neurology patients, the loss of smiling on command stems from a motor-cortex lesion, and that of spontaneous smiling from a subcortical one. Moreover, in Japan and England smiles are more regulated than in Latin America. But cultures everywhere show spontaneous smiling in greeting. Likewise, if I say that grieving the loss of a loved one is part of human nature, I refer to similar evidence. Neither individual exceptions nor cultural rules of expression undermine the hypothesis that it is human nature to grieve.

Paul Griffiths redefines emotions as phylogenetically shared affect programs. This makes them likely to be genetically guided nervous-system functions, beyond arousal or learning. Darwin's example of this, blushing, is unique to humans but physiologically biased and phylogenetically based. Some cognitive features of mind could be similarly defined; others wouldn't stand the test of shared phylogeny. This is true of emotion too.

It would be disingenuous to omit hatred, lust and greed, which come to mind most readily when human nature is mentioned. Why can't we calmly try to find out just how deeply ingrained they are? In the era of genomics and brain imaging, hypotheses about human nature are more testable than ever. Must we take offence at every potential limit that biology may place upon our freedom? Or can we take comfort that there are some that we all share? Only if human nature exists can we truly say that to gaze at a stranger's face is like looking in a mirror, and one that reflects things behind the face.

Melvin Konner, who teaches at Emory University, Atlanta, Georgia, USA, is the author of the revised edition of The Tangled Wing: Biological Constraints on the Human Spirit.

\section{FURTHER READING}

Darwin, C. The Expression of the Emotions in Man and Animals 3rd edn (0xford Univ. Press, 0xford, 1998). Eibl-Eibesfeldt, I. Human Ethology (Aldine de Gruyter, New York, 1989).

Ekman, P. \& Rosenberg, E. L. (eds) What the Face Reveals: Basic and Applied Studies of Spontaneous Expression Using the Facial Action Coding System (Oxford Univ. Press, Oxford, 1997).

Griffiths, P. What Emotions Really Are: The Problem of Psychological Categories (Univ. Chicago Press, Chicago, 1997).

Greenberg, J. et al. (eds) Universals of Human Language (Stanford Univ. Press, Stanford, 1978). 